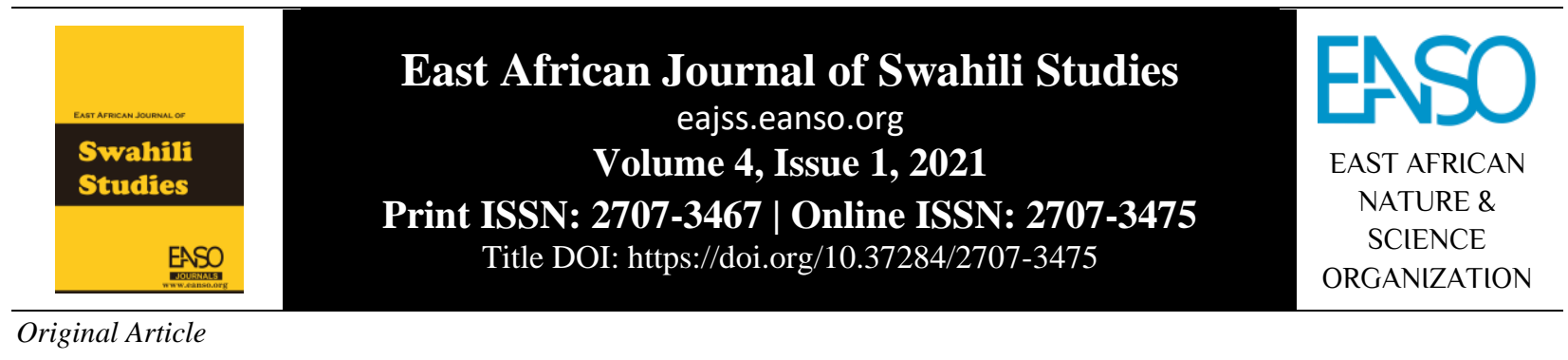

\title{
Utumikizi wa Mwingilianotanzu katika Fasihi ya Watoto.
}

Caroline Nyambura Muriithi ${ }^{1 *} \&$ Dkt. Boniface Ngugi ${ }^{2}$

${ }^{1}$ Chuo Kikuu cha Mlima Kenya, S.L.P 342-01000, Thika, Kenya.

*Barua pepe ya mawasiliano: mugocarol2011@gmail.com.

DOI ya Nakala: https://doi.org/10.37284/eajss.4.1.439

Tarehe ya Uchapishaji: IKISIRI

15 Oktoba 2021 Tanzu zimeendelea kujidhihirisha katika umbo la riwaya na kuifanya mseto wa mambo mbalimbali. Kutegemeana na kuathiriana kwa tanzu kunaitwa

Istilahi Muhimu: mwingilianotanzu. Fasihi ya watoto imeendelea kukua na kudhihirisha matumizi ya tanzu mbalimbali katika umbo lake na hivyo kuwa changamano.

Fasihi ya Watoto; Uchangamano huu unatatiza upokeaji na uelewekaji wa dhima yake, hasa

Mwingilianotanzu, ikizingatiwa kuwa riwaya hizo zinatumika katika mtaala wa elimu nchini.

Riwaya, Makala haya yananuia kufafanua ni vipi mwingilianotanzu unavyojisawiri

Habari zawio, katika vitabu vya fasihi ya watoto. Mifano itatokana na baadhi ya vitabu teule vya fasihi ya watoto ni Habari za Mawio (Masoud Nassor) na Maskini Bibi

Maskini Bibi Yangu. Yangu! (Said A Mohamed). Mwishowe itapendekeza namna mwingilianotanzu unavyoweza kutumiwa kwa ufaafu zaidi katika uelekezaji wa usomaji na kuuwasilisha ujumbe katika fasihi ya watoto.

\section{APA CITATION}

Muriithi, C. N. \& Ngugi, B. (2021). Utumikizi wa Mwingilianotanzu katika Fasihi ya Watoto. East African Journal of Swahili Studies, 4(1), 1-11. https://doi.org/10.37284/eajss.4.1.439.

\section{CHICAGO CITATION}

Muriithi, Caroline Nyambura \& Boniface Ngugi. 2021. "Utumikizi wa Mwingilianotanzu katika Fasihi ya Watoto". East African Journal of Swahili Studies 4 (1), 1-11. https://doi.org/10.37284/eajss.4.1.439.

\section{HARVARD CITATION}

Muriithi, C. N. \& Ngugi, B. (2021) “Utumikizi wa Mwingilianotanzu katika Fasihi ya Watoto”, East African Journal of Swahili Studies, 4(1), pp. 1-11. doi: 10.37284/eajss.4.1.439.

\section{IEEE CITATION}

C. N. Muriithi, \& B. Ngugi, "Utumikizi wa Mwingilianotanzu katika Fasihi ya Watoto", EAJSS, vol 4, no. 1, pp. 1-11, Oct. 2021.

\section{MLA CITATION}

Muriithi, Caroline Nyambura \& Boniface Ngugi. "Utumikizi wa Mwingilianotanzu katika Fasihi ya Watoto". East African Journal of Swahili Studies, Vol. 4, no. 1, Oct. 2021, pp. 1-11, doi:10.37284/eajss.4.1.439.

1 | This work is licensed under a Creative Commons Attribution 4.0 International License. 


\section{UTANGULIZI}

Waandishi wa fasihi ya watoto hawajaachwa nyuma katika kutumia mwingilianotanzu kama mbinu inayowasilisha ujumbe kwa watoto. Mwingilianotanzu hurejelea namna utanzu unavyotagusana na tanzu zingine na matini yote kijumla ili kuboresha uwasilishaji wa ujumbe. Kulingana na Mulokozi (1989) hakuna utanzu wowote ule uliojitosheleza ila tanzu huingiliana na matumizi ya tanzu zingine ili kufaulisha uwasilishaji ujumbe.

Aidha Mulokozi anaendelea kusisitiza kuwa mojawapo ya sifa kuu ya fasihi ni vipera vyake kuingiliana katika utendaji. Kulingana na Kristeva (1980), hakuna matini iliyohuru. Kila matini inahusiana na kutegemea mseto wa matini nyingine. Mwingilianotanzu ni uchanganyaji wa vipengele mbalimbali vya fasihi. Kwa mfano mwandishi anaweza kuchopeka nyimbo, mashairi, hadithi ndani ya hadithi, hotuba na taarifa za vyombo vya habari ili kugusa hadhira yake barabara.

Makala hii inafafanua namna watunzi wa fasihi ya watoto wanavyotumia mwingilianotanzu kama mbinu na mtindo katika kuibua hisia, ubainishaji wa ujumbe na ujengaji wa muktadha elekezi katika usomaji wa vitabu vya fasihi ya watoto. Miongoni mwa watafiti kuhusu mwingilianotanzu ni Marete (1994); Kehinde (2003); Wamitila (2003a); Wanyela (2007) na Habwe (2010). Katika makala hii, mifano itatokana na baadhi ya vitabu teule vya fasihi ya watoto: Habari za Mawio kilichoandikwa na Masoud Nassor na Maskini Bibi Yangu! cha Said A. Mohamed.

\section{Fasihi ya Watoto}

Fasihi ya watoto ni sanaa iliyoandikwa ikiwalenga watoto au ikilenga hadhira ya watoto. Kwa mujibu wa katiba ya Kenya kifungu cha 260 , mtoto ni mtu wa chini ya miaka kumi naminane kwa sababu hajapevuka kiakili. Aidha hana ruhusa ya kuolewa na kuoa au uhuru wa kupiga kura. Fasihi ya watoto imepuuzwa sana na ni katika karne ya kumi na nane ambapo ilianza kutambuliwa na kupata umaarufu ambao umechangia kuchapishwa na kutathiminiwa kwa fasihi hii kulingana na Mlacha (1985). Katika juhudi za kuifanya fasihi hii kukua na kuwa na mashiko katika ulimwengu wa fasihi, mbinu mbalimbali zimetumiwa ili kuvutia hadhira. Mojawapo ya mbinu hizi ni matumizi ya mwingilianotanzu. Ufafanuzi huu ni awa muhimu kwa vile riwaya zilizoteuliwa ni za watoto wa umri kati ya tisa hadi kumi na minne. Kadhalika mbinu ya mwingilianotanzu imetumika kwa mapana na marefu katika kufanikisha ujumbe wa waandishi kwa hadhira.

\section{Mwingilianotanzu}

Mwingilianotanzu hurejelea utanzu ulivyotagusana na tanzu zingine na matini yote kijumla ili kuboresha uwasilishaji wa ujumbe. Mulokozi (1989) anasema kuwa hakuna utanzu wowote ule uliojitosheleza ila tanzu huingiliana na matumizi ya tanzu zingine ili kufaulisha uwasilishaji wa ujumbe. Kulingana na Bakhtin (1982); Kristeva (1980); Barthes (2002 na 2007); Bloom (1994) na Foucait (1974) mtindo wa tanzu au matini kutegemeana na kuingiliana ndio unaoitwa mwingilianotanzu, Kwa mujibu wa wataalamu hawa mwingilianotanzu ni uumbaji wa matini kutokana na matini nyingine.

Matumizi ya mwingilianotanzu yamedhihirika katika vitabu vya fasihi ya watoto na kuchangia katika kuongezeka kwa wasomaji wa kazi za watoto jambo ambalo limefanya fasihi ya watoto iendelee kukua na kupanuka katika tanzu zake na kupata mwamko mpya. Makala haya yalishughulikia namna mwingilianotanzu ulivyo jitokeza katika riwaya teule za fasihi ya watoto; Habari za Mawio (Masoud Nassor) na Maskini Bibi Yangu! (Said A Mohammed)

Matumizi ya mwingilianotanzu yatahakikiwa kwa mujibu wa Wamitila (2002, uk. 153) na Bakhtin (1982) wanaoamini kuwa utanzu wa riwaya una uwezo wa kujumulisha wasifu wa tanzu nyingine lakini ukabakia na sifa zake kama utanzu. Kwa mfano riwaya inaweza kuhusisha mazungumzo, hadithi ndani ya hadithi, taarifa za vyombo vya habari na ushairi na bado ikabakia riwaya.

\section{MSINGI WA NADHARIA}

Nadharia hii ina misingi yake katika nadharia ya usemezano iliyoasisiwa na Mikhail Mikhailovich Bakhtin (1982) aliyedai kuwa matini ya kisemezano inaendeleza usemezano na matini nyingine za fasihi na hata watunzi. Matini hiyo inajibu, inasahihisha, inanyamazisha na kuendeleza matini za awali.

2 | This work is licensed under a Creative Commons Attribution 4.0 International License. 
Mishra (2012) anashadidia haya kwa kusema kuwa waasisi wa Nadharia ya Mwingilianomatini ni Ferdinand de Aassure, Pierce, Eliot na Bakhtin. Kisha iliendelezwa na Julia Kristeva, Ronald Barthes, Harold Boom na Gerald Gennette. Kristeva anadai kuwa waandishi hawatungi matini zao kutoka katika mawazo yao, bali kutokana na matini za awali. Hivyo basi Kristeva anaamini kuwa mwingilianomatini ni hali ambapo matini inadhihirisha usomaji mseto wa matini za awali, hivyo kuielewa matini na kujibu mwingine.

Wanamwingilianomatini wengine kama Bloom (1994) na Barthes (2002) wanaamini kuwa mwingilianomatini ni usukaji wa maana ya matini kutokana na matini nyingine. Nadharia hii inakumbusha kuwa kila matini inahusiana na matini nyinginezo. Mihimili ya mwingilianotanzu iliyotumika ni kama ifuatayo; Mosi, Waandishi hutunga matini zao kutokana na matini za awali na wala hawaziandiki kutoka katika mawazo yao. Kutokana na hili, matini zinahusisha kauli na tanzu kutokana na matini nyingine za awali na za sasa. Hivyo basi, matini zinaingiliana na kuathiriana pakubwa. Pili, maana ya matini haipatikani katika matini yenyewe bali inajengwa na msomaji katika matini husika na mseto wa matini nyingine zinazotajwa na kurejelewa katika usomaji huo. Tatu, matini ya kifasihi inaweza kujidhihirisha au kujihusisha na matini nyingine kiwaziwazi, kwa kunukuu, kudondoa, kutaja, kurejelea au kusimilisha sifa za matini za awali; au kuwa katika mkumbo mmoja wa kifasihi

Ni muhimu kutaja kwamba, katika utafiti huu, si mihimili yote ambayo ilitumika. Iliyotumika ni ile tu, iliyofaa zaidi kufafanua riwaya zilizochunguzwa ili kuweza kuangazia dhana ya mwingilianotanzu. Hivyo basi, nadharia hii ya mwingilianomatini ilisaidia katika ukusanyaji, uwasilishaji na uchanganuzi wa data.

\section{MBINU ZA UTAFITI}

Mbinu ya kuteua sampuli katika utafiti huu ilikuwa ya mtindo wa sampuli makusudi. Mtafiti aliteua riwaya za watoto kwa kutumia sampuli makusudi. Riwaya hizi ni sehemu ya riwaya arobaini (40) zilizokusanywa zenye data mwafaka na ni baadhi ya miongoni mwa zile ambazo zilipendekezwa na kupitishwa na ile iliyokuwa Taasisi ya Elimu nchini
Kenya (KIE) sasa (KICD) kusomwa na wanafunzi katika darasa la nne hadi la nane na ziliweza kupatikana kwa urahisi kwa lengo la utafiti. Mtafiti alitumia mbinu ya utafiti uchanganuzi wa yaliyomo kama utaratibu wa utafiti wa maandishi. Mbinu hii huwa mfumo wa kuchunguza kwa utaratibu yaliyoandikwa katika vitabu. Maktabani mtafiti alisoma maandishi ambayo yaligusia mwingilianomatini na jinsi ulivyotumika kama mbinu ya uandishi kupitia hadithi ndani ya hadithi, mazungumzo, taarifa za vyombo vya habari, ushairi na nyimbo na amatumizi ya gazeti.

Baadhi ya mihimili yake ndiyo iliyotumiwa katika uchanganuzi wa data iliyokusanywa. Jumla ya vitabu viwili vya fasihi ya watoto vilivyoteuliwa vilitumiwa kupata data iliyohitajika katika uchanganuzi.

\section{MATOKEO YA UTAFITI}

Matokeo ya utafiti huu yaliwasilishwa kwa njia ya maelezo. Data iliyokusanywa ilichanganuliwa kwa kuzingatia malengo ya utafiti, sababu za utafiti na nadharia iliyoongoza utafiti. Baada ya kunakili yaliyomo, data ilichanganuliwa. Dhana zilizogusia mwingilianotanzu zilitambuliwa. Matumizi ya mwingilianotanzu kulingana na nadharia ya mwingilianomatini yaliwekwa wazi kwa kutegemea riwaya mbili teule za fasihi ya watoto.

\section{Ushairi na Nyimbo}

Harries (1962, uk. 272) anasema “....wimbo ni shairi ndogo. Shairi ni wimbo mkubwa na utenzi ni upeo wa shairi." Wamitila (2003) anaongeza kwa kufafanua kuwa ushairi ni utungo ambao una mistari iliyogawika katika mafungu kadhaa na unaweza kuwa na mpangilio unaoonyesha mfuatano maalum ya silabi au ukawa hata na idadi maalumu ya silabi hizo. Pia, inawezekana mpangilio wake ukawa huru. Anaendelea kusisitiza kuwa wimbo ni bahari mojawapo ya ushairi.

Mulokozi (1996) anasema kuwa wimbo ni kila kinachoimbwa. Anaendelea kueleza kuwa mambo muhimu katika nyimbo ni muziki wa sauti ya mwimbaji, matini au maneno yanayoimbwa, na muktadha unaofungamana na wimbo huo.

Katika riwaya ya Habari za Wawio (Masoud Nassor) na Maskini Bibi Yangu! (Said A 
Mohammed) ushairi umejitokeza. Kwenye riwaya ya Habari za Mawio kuna Shairi Waridi Lisilo Miba.

Ewe kiumbe wa shani, nalipenda umbo lako,

Mzuri uso kifani, kakuumba Mola wako,

Katu humu duniani, hapana mfano wako,

Ewe ua la peponi, waridi lisilo miba (uk. 13).

Hili ni shairi lake Isa Matono ambaye ni gwiji wa taarabu. Katika mwanzo na mloto wa shairi hili malenga anasifu urembo usiomithilika kutokana na uumbaji wa aina yake na Mola. Urembo huu unazidi kusifiwa katika mlea na kimalizio. Shairi hili linaibua maudhui ya mapenzi kwa vile linaelekezwa kwa mtu fulani anayeenziwa kutokana na maumbile yake ya kuvutia na yasiyolinganishika. Bakhtin (1968) akieleza kuhusu mwingilianotanzu anasema kuwa matini huweza kuingiza sifa za tanzu zingine na maumbo asilia na kuyabadilisha na kuyajenga upya. Vilevile katika makala 'The problem of speech genres", Bakhtin (1986) anatofautisha tanzu sahili na tanzu changamano. Anaeleza kuwa tanzu changamano kama riwaya na tamthilia 'humeza na kuyeyusha'tanzu sahili kadhaa ambazo hubadilishwa ziingiapo katika tanzu changamano. Katika kazi hii ushairi ni utanzu sahili unaomezwa na kuyeyushwa na riwaya inayochukua nafasi ya tanzu sahili.

Aidha kuna ushairi wa Siti binti Saad (1880-1950).

\section{Sitaki sitaki kuliliwa mwao,}

Toka vijakazi na vibibi vyao,

Walilia ndizi mgomba si wao (uk. 14).

Sinti binti Saad alikuwa gwiji wa taarab miaka ya ishirini. Mwimbaji huyu alimfanya Shabaan Robert kuandika kuhusu maisha ya msanii huyu mashuhuri kwa jinsi sifa zake zilivyoenea. Katika wimbo huu anasema kuwa hataki maisha yake kuingiliwa kwani hawayahusu wengine. Kadhalika kulingana na wimbo huu alilenga kutenda haki kwa kujali vizazi vijavyo. Ushairi huu ulinukuliwa na Msamaria kwa Ali wakiwa katika kibanda cha Mamalishe. Katika kunukuu huku Msamaria alikuwa akitaka kuonyesha weledi wake kwa nyimbo za Taarab. Kulingana na Msamaria shairi la mwimbaji huyu lilimfanya Shabaan Robert kuandika kuhusu maisha ya msanii huyu mashuhuri.

Riwaya ya Maskini Bibi Yangu! kuna wimbo wa mashabiki kwa Chita wakimshangilia.

\section{Chita Chita chita}

\section{Chita hakuna wasiwasi}

Chata hakuna wasiwasi (uk. 20).

Wimbo huu umenukuliwa katika kisa ndani ya kisa kwa Ajili ya Bi. Mbunjaki. Mashabiki wa Chita walikuwa wakimshangilia katika mashindano ya kitaifa ambayo alihiari kushiriki kwa Ajili ya azma yake ya kutaka kurudisha nuru ya Bi. Mbunjaki.

\section{Taarifa za Vyombo vya Habari katika Riwaya}

Watunzi wa Habari za Mawio (Masoud Nassor) na Maskini Bibi Yangu! (Said A. Mohammed) wametumia vyombo vya habari vikiwemo magazeti na redio kama mbinu mojawapo ya mwingilianotanzu. Kulingana na mihimili ya mwingilianomatini hakuna matini inayojitenga na nyingine. Bloom (1994) na Barthes (2002) wanaamini kuwa mwingilianomatini ni usukaji wa maana kutokana na matini nyingine. Kila matini inahusiana na matini nyinginezo.

Katika Habari za Mawio (Masoud Nassor), vyombo vya habari, hasa redio na magazeti vimetumika. Kuna kituo cha sauti ya Tanzania, Zanzibar ambacho kinatangaza habari kwa watu wanaoishi visiwani Unguja na Pemba. Maelezo ya habari yanatolewa na msimulizi aliyekuwa kwenye boti akielekea Unguja.

Hizi ni Habari za Mawio na penye mawio utasikia habari za Seastar na kwenye Seastar utapata Habari za Mawio (uk. 1).

Vilevile kuna mazungumzo baada ya habari yaliyopeperushwa kupitia Redio Tanzania, Dar es Salaam. Mazungumzo yalieleza kuhusu ugonjwa wa ukimwi:

Watu wasipojichunga na Ukimwi, utawamaliza na matokeo yake ... taifa hili litaangamia pia. Wakati umefika wa watu kujiuliza; kupi bora; kufa kwa gonjwa hili la hatari na la aibu au

4 | This work is licensed under a Creative Commons Attribution 4.0 International License. 
kuishi kwa heshima na salama? Chaguo ni lako (uk. 1).

Huu ulikuwa mwisho wa mazungumzo baada ya habari yaliyopeperushwa kupitia Redio Tanzania Dar es Salaam. Kutokana na mazungumzo haya ni kweli kuwa hakuna usomaji wa matini ambao si utunzi mpya. Kwa mujibu wa Eagleton (1983) maana ya matini haipatikani katika matini yenyewe, bali inajengwa na msomaji katika matini husika na mseto wa matini nyingine zilizotajwa na kurejelewa katika usomaji huo.

Isitoshe kuna matumizi ya vyombo vya habari katika harakati za kuelimisha umma kuhusu ugonjwa wa Ukimwi.

\section{"Ufuatao ni Muhtasari wa habari."}

Mtangazaji akasema; "Serikali imeamua kupiga marufuku magazeti na vijarida kadhaa vinavyochapisha picha za watu wakiwa nusu uchi. Wataalamu wa saikolojia wa chuo kikuu cha Nairobi wanasema kuwa kutokana na tafiti mbalimbali zilizofanywa, inathibitishiwa kuwa picha hizo zinazochapishwa na magazeti hayo zinachangia kuibua hisia za ngono, hasa miongoni mwa vijana, na hivyo basi kuchangia ueneaji wa magonjwa ya zinaa kusababisha kuenea pia kwa UKIMWI (uk. 22).

Huu ulikuwa muhtasari wa habari uliopeperushwa moja kwa moja na kituo cha Sauti ya Tanzania Zanzibar. Mtangazaji aliyetangaza alikuwa na dhima ya kuwatahadharisha watu kuhusu ugonjwa wa Ukimwi. Kati ya wasikilizaji alikuwa Ali ambaye hakuyaelewa hata kidogo maana ya maudhui katika muhtasari wa habari.

Baada ya mazungumzo ya Ali na Msamaria kumhusu Musa, kakake Ali kulikuwa na matangazo kutoka Sauti ya Tanzania, Zanzibar.

Kaka yake msanii maarufu wa Jitambue kwa jina la Musa Masudi aliyekuwa amelazwa katika Hospitali ya Mnazi Mmoja, amefariki dunia baada ya kuugua kwa mwezi. Sauti ya Tanzania, Zanzibar inampa pole kijana msanii na mwelimishaji Ali Masudi kwa msiba huo wa kaka yake Mungu ailaze pema roho ya Marehemu. Amin! (uk. 42).
Matangazo haya yalitolewa siku ya tatu tangu Msamaria wazungumze na Ali kuhusu habari za ndugu yake Musa katika kituo cha Jitambue.

\section{Gazeti}

Gazeti kulingana na kamusi ya Kiswahili Sanifu Toleo la Pili ni karatasi zilizochapishwa habari na kutolewa mara kwa mara ama kila siku au wiki kwa lengo la kuelimisha au kufahamisha umma kuhusu mambo mbalimbali. Gazeti imejitokeza kama mbinu muhimu katika kazi ya Masoud Nassor. Baada ya kifo cha mwimbaji taarab Mashuhuri Isa Matona mnamo mwaka 2004 gazeti moja liliripoti kifo chake;

Hasara kubwa ilioje katika ulimwengu wa sanaa kwa kuondokewa na mwana huyu mweledi wa fani ya taarab (uk. 13).

Katika tangazo hili maudhui ya kifo yaliwekwa wazi na vilevile ukiwa ulidhihirika kwa wapenzi wa Taarab kutokana na kuondokewa na shujaa huyu wa Taarab.

\section{Hadithi ndani ya Hadithi}

Hadithi ndani ya hadithi ni dhana inayomaanisha simulizi moja kuingiliwa na nyingine. Kwa mfano msimulizi mkuu anampa nafasi mhusika katika hadithi kusimulia hadithi nyingine. Katika dhana hii kunakuwa na hadhira tofauti kutokana na idadi ya usimulizi. Mathalani, tunaposoma hadithi halafu ndani ya hadithi hiyo tukakuta mhusika mwingine ndani ya hadithi hiyo akiwasimulia wengine, hapo tunakuwa na hadhira ya aina mbili. Sisi kama wasomaji tunakuwa hadhira na wale wanaosimuliwa. Hadithi ndani ya hadithi ni kipengele kingine cha fasihi simulizi kinachojitokeza katika kazi ya Masoud Nassor; Habari za Mawio. Njogu na Chimera (1999) wanaeleza kuwa usimulizi wa ngano huwa ni wa moja kwa moja hata hivyo hujumuisha mahojiano, malumbano au hadithi ndani ya hadithi husika. Mbinu hizi hutumika ili kueleweka kwa maudhui kwa urahisi. Eagleton (1983) anasema kuwa kazi zote za fasihi huandikwa upya kwa kiwango fulani, ingawa bila ya jamii husika kufahamu. Kwake anasisitiza kuwa hakuna usomaji wa matini ambao si utunzi mpya. Kulingana naye matini ya kifasihi inaweza kujidhihirisha au kujihusisha na matini nyingine kiwaziwazi, kwa kunukuu, kudondoa,

5 | This work is licensed under a Creative Commons Attribution 4.0 International License. 
kutaja, kurejelea au kusimilisha sifa za matini za awali; au kuwa katika mkumbo mmoja wa kifasihi.

Katika riwaya ya Habari za Mawio kuna Kisa cha Idrisa mtoto wa Feri.

Kisa hiki ni mfano wa ngano za usuli kwani kinaeleza chanzo cha tabia ya Idrisa kupenda kwenda Feri. Inasemekana wazazi wake walikufa baada ya kuzama kwenye jahazi lililokuwa karibu likikaribia kutia nanga. Alipopata fahamu tu, alianza kuja Feri na kuangalia majahazi yaliyokuwa yakija kwa matumaini ya kuwaona wazazi wake wakitoka jahazini (uk. 24).

Hiki ni kisa cha Idrisa ambaye alikuwa akifanya kazi ya kupara samaki. Nshazume alimtambia Ali hadithi hii katika harakati zao za kila siku za kupara samaki kwenye feri kwa lengo la kumpa matumaini. Kijana huyu kwa jina Idrisa alikuwa amepitia changamoto nyingi katika maisha yake lakini alikuwa amezipiku na kuanza kujitegemea maishani.

Kadhalika katika riwaya ya Habari za Mawio kuna kisa cha Nshazuma kutengwa na wenzake. Wamitila (2002, ik. 153), Bakhtin (1982) wanaamini kuwa utanzu wa riwaya una uwezo wa kujumulisha wasifu wa tanzu nyingine lakini ukabakia na sifa zake kama utanzu;

Nshazume alikuwa na mchumba aliyempenda sana lakini kabla yake kumиoa, fununu zikaenea kuwa mchumba aliyempenda sana lakini kabla yake kuтиоа, fununu zikaenea kuwa mchumba wake alikuwa mjamzito ujauzito ulikuwa wa Nshazume. Kila mtu akamuelekezea kidole cha lawama japo hakuwa amehusika kamwe. Ingawa alikuwa anampenda sana mchumba wake hangeweza kumuoa kutokana na umaskini. Hivyo akaingia jahazini ili aweze kutokomea bara. Lakini lo! Upepo ukapiga jahazi nusura izame ... Basi mganga maarufu akamtaja kuwa ndiye mwenye nuksi mle jahazini. Watu wote wakataka kumtosa baharini kama vile Nabii Yunus lakini akanusuriwa na msamaria. Tangu siku hiyo Nshazume hawezi kurudi kwao kwa kuwa hakuna jahazi linaloweza kumvua kwa kuaminiwa kuwa ni mwenye nuksi (uk. 26).
Nshazume alikuwa akitamba kisa hiki kwa rafiki yake Ali kwenye feri. Kwenye kisa hiki Imani za kishirikina zinadhihirika kupitia kwa watu kwa watu kwenye feri na kwenye jahazi. Hata hivyo ukarimu na utu wa Msamaria unaonekana wakati alimwokoa Nshazume.

Kadhalika kuna hadithi ya kimafumbo iliyosimuliwa kwa Ali na Msamaria. Hadithi hii ilikuwa ya kutoa mwanga maana kuwa ujana ni wakati wa vitendo na uzee ni wakati wa kumbukumbu.

Katika hadithi hii kulikuwa na wagonjwa wawili Badi na Juma waliokuwa wamelazwa kwenye wadi moja hospitalini. Wagonjwa hawa waliunga urafiki kwa vile ugonjwa uliwaunganisha. Walichukua fursa ya kujikumbusha mengi ya ujana wao. Badi alikuwa akikaa karibu na dirisha la pekee katika wodi hiyo. Hivyo basi Badi alimweleza Juma kilichokuwa kikipita nje kwani alikuwa hodari katika kuhadithia. Siku moja nesi alipofika kuleta kisabeho alijaribu kumwamsha Badi lakini alikuwa keshaaga. Siku ya pili, Juma akamwomba nesi amsogezee kitanda chake karibu na dirisha kwani alikuwa na kiu cha kuuona ulimwengu aliokuwa akielezwa na marehemu Badi. Ila alipoangalia, alikuta kuwa dirisha lile lilikuwa likiangaliana na ukuta wa jengo jingine. Akaona kama alisalitiwa kwa kuelezwa taswira za hekaya. Hatimaye nesi alimwambia Juma kuwa Badi alikuwa kipofu, hata ule ukuta alikuwa hauoni! Nesi alimweleza Juma kuwa nia ya Badi ilikuwa kumtia moyo na kumpa matumaini. Yaani, kuwa hata katika ugonjwa na mauti, bado tunaruhusiwa kuwa na matumaini na kutegemea mafanikio mema na furaha... (uk. 38).

Hiki ni kisa cha Msamaria kwa Ali wakiwa kwenye feri walikokuwa wakifanya kazi. Msamaria alikuwa mzee mwenye busara na katika kisa hiki alijaribu kumwonyesha Ali umuhimu wa kuwafariji watu wenye huzuni ili kuwapa furaha hata kama wewe mwenyewe pengine una majonzi na shida.

Kuna kisa cha Mkojani aliyefukuzwa na ng'ombe aliyekata kamba. Mkojani huyo aliyekuwa akikimbia alimkuta mwenziwe bondeni alikisali. Akamwambia kuwa huko nyuma kuna ng'ombe aliyekata kamba na

6 | This work is licensed under a Creative Commons Attribution 4.0 International License. 
anafukuza na kuwapiga watu pembe. Kawaida mtu akisali huwa hasemi na yeyote ila Mwenyezi Mungu. Sasa akamwambia mwenziwe aатиe kusali au kuokoa maisha yake (uk. 18).

Hiki ni kisa kilichosimuliwa na Hamis kwa Ali kuhusu Wakojani. Kisa hiki kililenga kueleza Ali kwa nini Nshazume ambaye alikuwa Mkojani alipigwa pande na wenzake. Masimulizi haya yalitokea sikunya kwanza Hamis alipofahamishwa na Msamaria kwa Ali. Dhima ya usimulizi huu ilikuwa kuonyesha usuli na msimamo madhubuti ya Wakojani.

Riwaya ya Said A. Mohammed, Maskini Bibi Yangu! Inazidi kudhihirisha utanzu wa hadithi ndani ya hadithi. Eagleton (1983) anasema kuwa maana ya matini haipatikani katika matini yenyewe bali inajengwa na msomaji katika matini nyingine zinazotajwa na kurejelewa katika usomaji. katika riwaya ya Maskini Bibi Yangu! Kuna kisa chenye mada kwa Ajili ya Bi. Mbunjaki. Kisa hiki kinajitokeza katika ndani ya kitabu hiki.

Bi. Mbunjaki alikuwa bibi mkongwe aliyezaliwa kabla ya kimbunga cha Zanzibar. Alikuwa mkunga aliyekula chumvi nyingi. Hakufanikiwa kuwa na watoto wake maalumu japo alikuwa bibi wa kila mtu na kila mtu alikuwa mjukuu, kitukuu, kilembwe, kilembwekeza au kinyinginya kwake. Kati ya watu waliopenda Zaidi alikuwa Daudi. Bibi huyu alikuwa kapotelewa na uwezo wa kuona na hivyo Daudi alijaribu juu chini kuhakikisha bibi huyu amerudiwa na uwezo wake wa kuona. Daudi alishiriki mashindano ya kukimbia kwa ajili ya Bi. Mbunjaki na akaibuka mshindi (uk. 12).

Hii ni hadithi inayosimuliwa na Daudi kuhusu Bi. Mbunjaki mwanamke ambaye alimpenda kwa dhati. Hadithi hii inaibua masuala ibuka mengi katika jamii na umuhimu wa ukarimu.

Kwa mujibu wa Wamitila (2002, uk. 153) na Bakhtin (1982) wanaamini kuwa utanzu wa riwaya una uwezo wa kujumulisha wasifu wa tanzu nyingine lakini ukabakia na sifa zake kama utanzu.

\section{Mazungumzo}

Mazungumzo ni mojawapo ya tanzu za fasihi simulizi. Kulingana na Assumta Matei (2011) mazungumzo ni utaratibu wa kuongea baina ya pande mbili tofauti kuhusu mada mbalimbali zinazobadilikabadilika ambapo kwa kawaida wahusika wake huwa ni binadamu. Mazungumzo ya kifasihi yanaweza kuainishwa katika vipera vya malumbano ya utani, mawaidha, soga na hotuba. Vipera hivi vimetumika katika riwaya mbili teule kufanikisha mwingilianotanzu. Mulokozi (1989) anasema kuwa hakuna utanzu wowote ule uliojitosheleza ila tanzu huingiliana na matumizi ya tanzu zingine ili kufaulisha uwasilishaji wa ujumbe.

Katika riwaya ya Habari za Mawio na Maskini Bibi Yangu! kipera cha malumbano ya utani kimetumika kufanikisha mwingilianotanzu.

\section{Utani wa Maumbu}

TUKI (2004, uk. 446) inafafanua dhana hii ya utani ikisema ni taratibu za kimila ambazo zinawafanya watu kuambiana au kutendeana jambo lolote bila chuki. Kyando (1993) anaeleza kwamba masihara ni sehemu ndogo tu ya utani. Na utani ni mahusiano yanayojengeka katika jamii yenye lengo la kukuza ushirikiano na mahusiano baina ya koo, kabila na familia, ambapo watu huweza kuambiana neno lolote au kuchukuliana vitu bila kuleta athari yoyote. Aidha, Haji (1992) anaueleza utani kwamba ni mpango maalumu unaofuatwa kimila ambao unawafanya watu kuambiana au kutendeana jambo lolote bila chuki. Zaidi, ni kuambiana na kutendeana mambo ya mzaha, dhihaka na bila kuleta taathira yeyote. Naye Sengo na Lucas (1975, uk. 14) wanafafanua dhana hii kuwa ni neno la Kiswahili lihusulo mwenendo au utaratibu wa maisha unaoingiliana na mila na desturi kadha wa kadha katika jumuiya za wenyeji wa Afrika Mashariki.

Akinukuu kamusi la American Heritage, Mzandi (2011, uk. 32) anasema:

Jokes are an amusing story with punch, with an affective but incising message. However the message and its presentation is not intended to harm anybody. In other words, a joke is a prank or jest. Jokes are usually between peoples with a special relationship that permits one to tease another without taking offence.

Utani ni hali ya uchekeshaji ambao ndani yake kuna ujumbe maalum uliokusudiwa kwa mtu kuupata. Hata hivyo, tunaweza kusema ya kuwa

$7 \mid$ This work is licensed under a Creative Commons Attribution 4.0 International License. 
utani ni hali ya kufanyiana mzaha, dhihaka masihara na kutaniana. Kwa kawaida utani hufanywa na watu wenye uhusiano maalumu unaoruhusu watu kutaniana bila kukosana (Tafsiri ya Mtafiti).

Utani ni uhusiano wa kutaniana au kufanyiana masikhara ambao ni tofauti na kutokuheshimiana, na madhumuni yake makubwa ni kupunguza na kuondoa kabisa chuki iliyopo baina ya makundi fulani ya watu. Kwa kawaida utani hufanywa na watu wenye mahusiano ya kiutani japo kidogo na si rahisi kufanya utani kwa mtu usiyemjua. Hata hivyo, utani mara nyingine hutumika ili kumuomba mtu ridhaa ya kuwa mtani wako na asiporidhia basi kanuni haikulazimishi kuendelea kumtania mtu huyo.

Kulingana na Kamusi ya Kiswahili utani ni majibizano kati ya watu wawili au makundi mawili kwa nia ya kutaniana. $\mathrm{Ni}$ mazungumzo yanayofanywa kwa nia ya kutaniana. Utani wa maumbu ni utani wa ndugu wazawa wa kike au wa kiume. Mazungumzo haya hufanywa kwa kutumia maneno ya mzaha ili kuleta ucheshi.

\section{Katika Habari za Mawio:}

Uk. 1, ndugu zake Ali, Fadhili na Musa walimtania Ali na kumwita 'Mtoto wa Mama'. Chuoni 'Ali mama' na mpirani akaitwa 'Mama'. Ali alizaliwa wakati mamake hakuwa na tamaa ya kuzaa tena. Jina hili la utani lilimkera japo hakuwa na hiari. Musa naye aliitwa kwa jina la utani 'Firauni' na Fadhili akaitwa 'Fedhuli' kutoka na ujeuri wake. Bakhtin (1982) anadai kuwa matini yoyote ile inahusiana na muktadha wa kitamaduni au kijamii ulioizalisha na haiwezi kutenganisha nao. Hivyo basi, matini zote zimesheheni imani na mawazo yanayotokana na miktadha hii.

\section{Utani wa Makabila.}

Utani wa makabila ni mazungumzo ambayo hufanywa na makabila ili kuchangamsha na kupunguza kiwango cha urasmi kati ya makabila. Utani huu hukuza utangamano miongoni mwa watu na makabila mbalimbali. Wakati mwingine utani wa makabila hukosoa na kukashifu tabia hasi katika makabila fulani kama vile ulafi, uchoyo na tabia nyingine mbaya.
Katika Habari za Mawio kuna utani mwingi kuhusu utamaduni wa watu wa visiwani na mwambao wa Afrika Mashariki. Wakojani ni watu wa kisiwa kidogo cha Kojani huko Pemba ambao wanasifika kwa utani mwingi. Wao hulinganishwa na kabila la Wabajuni. Wakojani wanataniwa kuwa wao huvaa miwani ya jua ndani ya jumba sinema (uk. 17).

Utani huu ulijitokeza wakati Ali na Hamisi walikuwa wakizungumza. Hamis alikuwa akimrai Ali asije akafanya urafiki na Nshazume kisa na maana alikuwa Mkojani watu ambao walifanyiwa utani na makabila mengi.

\section{Utani wa Mabibi na Wajukuu}

Huu ni utani unaofanywa kwa kutoa nafasi kwa bibi kuwa karibu na wajukuu zake. Katika aina hii ya utani babu huthubutu kumwita mjukuu wake wa kike mke, na bibi huweza kumwita mjukuu wake wa kiume mume. Ogacho (1974) na Chogero (1975) wamezungumzia mahusiano ya utani baina ya babu, bibi na wajukuu zake na kusema kuwa; wajukuu wanaweza huwa huru kuuliza maswali na kujifunza mambo mbalimbali ya kimaisha ikiwemo uwajibikaji katika kazi, dawa za asili, utunzaji wa familia na masuala ya ndoa. Uhusiano wao wa kiutani huwa endelevu hata baada ya bibi au babu kufariki.

Katika Masikini Bibi Yangu! kuna utani wa bibi kwa mjukuu wake. Shibu anataniwa na nyanyaye Bi Shumbi kama mume wake naye Shibu akimrejelea kama mke wake. Wanapokutana Bi Shumbi anamkumbatia na kumwambia,

Karibu bwana wangu! Karibu, Mume wangu!" Utani huu unadhihirisha mapenzi ya nyanya kwa mjukuu wake. Shibu alihisi furaha kwa sababu ya kuitwa 'bwana' kwa mara ya milioni. Mtu gani mwingine angemwita yeye 'bwana au mume wangu'? Ndiyo, Shibu alijua kuwa huo ulikuwa utani wa bibi na mjukuu wake (uk. 4-8).

Kisa cha Bi. Mbunjaki kinaingiza utani ambapo anamtania msimulizi, Daudi kama kibwana chake.

Ilinibidi nitumie ufundi wangu wote. Ilinilazimu nimalize ukunga wangu wote-enh, Bwana Daudi, enh kibwana changu! (uk. 13).

$8 \mid$ This work is licensed under a Creative Commons Attribution 4.0 International License. 
Daudi alimpenda sana Bi. Mbunjaki ambaye alikuwa mkongwe na asiye na familia yake kindandaki. Na alitamani kumrudishia uwezo wake wa kuona.

\section{Utani wa Marika}

Huu ni utani wa watu katika hirimu moja. Wanarika mara nyingi hutaniana kwa lengo la kukuza umoja kati yao. Katika Maskini Bibi Yangu! Daudi alirejelewa kama Chita na wanarika wenzake kutokana na uwezo wake wa kukimbia kwa kasi. Daudi alikuwa mkimbizi na sifa za kushinda zilizowafanya wenzake kumpa jina la utani.

'Chita kwa miaka saba alikuwa mshindi katika nambari ya kwanza katika mashindano ya mbio ya masafa marefu shuleni mwao na mashindano ya shule zote za wilayani na mkoani mwao. Alipokuwa akikimbia wanafunzi wenzake walimshangilia wakiimba na kupiga makofi 'chita, chita, chita (uk. 20-21).

\section{Mawaidha}

Mawaidha ni mojawapo ya kijitanzu cha mazungumzo. Mawaidha ni mazungumzo ambayo hutolewa ili kumpa ushauri au nasaha kuhusu jambo fulani. Mawaidha hutoa mwongozo na maelekezo ya kukabiliana na changamoto katika maisha na jinsi ya kuhusiana na wanajamii. Barthes (2002) anadai kuwa waandishi hutunga matini zao kutokana na matini za awali na wala hawaziandiki kutoka mawazo yao. Kutokana na hili, matini zinahusisha kauli na tanzu kutokana na matini nyingine za awali na za sasa. Hivyo, matini zinaingiliana na kuathiriana pakubwa.

Katika Habari za Mawio (uk. 26-33) Mazungumzo ya Nshazume na Ali yanaeleza njia ambazo mtu huambukizwa Ukimwi, na mikakati bora ya kujizuia na umuhimu wa elimu kwa umma kuhusu ugonjwa wa Ukimwi.

Aidha katika kazi yake Masoud Nassor, Msamaria anampa Ali mawaidha kuhusu ujana. Anamwambia,

Ujana una raha zake na uzee pia una raha zake. Ujana ni wakati wa matendo. Aidha uzee ni wakati wa kumbukumbu. Vijana wanatakiwa wachote hekima kutoka kwa wazee ili wasirudie makosa makubwa yaliyofanywa na vijana wa zamani. Kwani athari zake ndizo hizo tunazoziona leo (uk. 37).

Haya ni mazungumzo kati ya Msamaria na Ali. Msamaria alikuwa akimweleza Ali kuhusu umuhimu wa vijana kutumia muda wao ifaavyo. Kulingana na Msamaria vijana wanafaa kutumia muda wao kufanya mambo ya maana. Msamaria anasema kuwa kila sekunde vijana wanafaa kujifikiria upya katika maisha yao. Thamani ya muda wowote iwe sekunde au nusu sekunde analinganisha umuhimu wake kwa mkimbiaji mbio aliyepata medali ya Olimpiki kwa kushinda kwa unyoya tu. Thamani ya dakika anaonyesha umuhimu wake kwa mtu aliyeachwa na treni kwa kuwa alichelewa kuingia ndani. Thamani ya saa moja waulize wanandoa waliokuwa hawajaonana siku nyingi jinsi wanavyoona muda unavyochelewa kufika. Thamani ya wiki muulize mhariri wa gazeti la kila wiki anaposhindwa kutoa nakala muhimu, hasara kuba anayoipata. Hatimaye thamani ya mwezi mmoja ni mfano wa mjamzito aliyeharibu mimba.

\section{Soga}

Assumpta Matei (2011) anasema kuwa soga ni mazungumzo yanayonuiwa kupitisha wakati. Katika Habari za Mawio kipera cha soga kimetawala hadithi nzima kwa lengo la kupitisha wakati. Wavuvi walipokuwa wakipara samaki waliendeleza soga.

\section{HITIMISHO}

Ni wazi kuwa mwingilianotanzu umejitokeza kwa mapana kupitia mbinu mbalimbali. Kwa mujibu wa makala haya ni bayana kwamba mwingilianotanzu ni dhana ambayo imechangia kama mbinu na mtindo wa kuibua hisi na kufikisha ujumbe kwa wanaolengwa. Matumizi ya mwingilianotanzu katika riwaya hizi yanafanya msomaji aelewe kwa haraka na kuondoa uchovu.

\section{MAPENDEKEZO}

Ningependekeza kwamba watafiti wengine washughulikie umuhimu wa matumizi ya vielelezo (picha/michoro) katika kazi ya fasihi ya watoto. Utafiti huu wa baadaye unaweza kutumia nadharia

9 | This work is licensed under a Creative Commons Attribution 4.0 International License. 
nyingine na achunguze nafasi ya vielelezo hivi katika kufaulisha uwasilishaji wa ujumbe kwa hadhira.

\section{MAREJELEO}

Akoliet, J. (1990). "Response and crtitism in children's Literature in Kenya. The case of Barbara Kimenye." M. A. Thesis Kenyatta University.

Bakhtin, M. (1982). The Dialogic Imagination: Four Essays. Mh. Michael Holquist. Tafsiri. Caryl Emerson na Michael Holquist. Austin: University of Texas Press.

Bakize, L. (2014) Utangulizi Wa Fasihi ya Watoto. Dar es Salaam. Moccony Printing Press. UK 24.

Barthes, R. (2007). 'From Work to Text. ' Katika B. Das na J. M. Mahanty (wah), . Literary critism: A Reading (uk. 413-420). New Delhi: Oxford University Press. UK 413-420.

Barthes, R. (2002). 'The Death of The Author'. Katika Philip Rice na Patricia Waugh (wah.). Modern Literary Theory. New York: Arnold. uk.185-189.

Bloom, H. (1994). The Western Canon. New York: Harcourt Brace and Company.

Cass, J. (1967). Literature and the Young Child. London: Longman Group Ltd.

Chogero, B. (1971), The Socio-Economic Implications of Utani in Jita Society. Dar es Salaam University Press.

Davies, A. 1973. Literature for Children. Portsmouth: Open University Press.

Eagleton, T. (1983). Literary Theory: An Introduction. Oxford: Basil Blackwell.

Finnegan, R. (1970). Oral Literature in Africa. Nairobi. Oxford University Press.

Foucault, M. (1974). The Archeology of knowledge. London: Tavistock.

Habwe, J. H. (2010). Dialogue drama in Kenyan political speeches and its pragmatic implications. Nordic journal of African studies, 19(3), 16-16. Habwe. J. (2010) 'Dialogue Drama in Kenyan political speeches and its pragmatic implication: katika Nordic Journal of African studies 19(3). Hersinki: Nordic Association of African Studies (NAAS). UK. 165-180.

Haji, M. (2006), Vipera vya Fasihi Simulizi, Central Publishing Services Tanzania, Zanzibar.

Harries. L. (1962) Swahili Poetrv. Oxford. Clarendon Press.

Herman, L. (2011). "Dhima ya mwingilianomatini kwenye Hadithi za watoto katika Kiswahili, " katika kioo cha lugha, Juz. 9, Kur. 73-83.

Hunt. P. (1994). An Introduction to Chidren's Literature. Oxford: University Press.

Jan, J. (1969). Children is Literature. London: Trinity Press.

Kehinde. A. (2003). 'Intertextuality and The Contemporary African Novel. Nordic Journal of African Studies, 12(3), 372-386' katika Nordic Journal of African Studies 12(3). Helsinki: Nordic Assoication of African studies (NAAS). UK 372-386.

Kothari, C. (2004). Research methodology: Methods and techniques. New Age International.

Kristeva, J. (1980). Desire in language: A Semiotic Approach to Literature and Art. New York: Columbia University press.

Kyando, A.S.J. (1973), The Kinga, URT, Vol.II, Dar es Salaam University Press.

Lukas, G. (1972). Studies in European Realism. A sociological survey of writings of Balzaac, Stredhal, Zola, Tolistoy, Govki and others. London: Marlin Press.

Marete, J. (1994). Ujadi katika tamthilia mbili za Emmanuel Mbogo. Tasnifu ya Uzimili (Haijachapishwa)

Matei, K.A. (2011). Fani ya Fasihi Simulizi kwa shule za upili. Nairobi. Oxford University

10 This work is licensed under a Creative Commons Attribution 4.0 International License. 
Mishra, R. K. (2012). 'A Study of Intertextuality: The Way of Reading and Writing'. Katika Prime Journals.

Mlacha, S. A. K. (1985). Kiswahili katika Kanda ya A Frika Mashariki. Dar es Salaam.

Wamitila, K. (2010). Kanzi ya Fasihi (1): Misingi ya uchanganuzi wa Fasihi. Nairobi: vide- Muwa Publisher Limited.

Mpesha, N. (1995). “Chidren's Literature in Tanzania: A Literary Appreciation of its Growth and Development." $\mathrm{PhD}$ Thesis, Kenyatta University.

Mulokozi, M. M. (2018). Tanzu za fasihi simulizi. Mulika Journal,21(1), 1-24. Mulokozi, M. (1989). "Tanzu za fasihi simulizi." Katika, mulika. Na 21. Dar es Salaam. Taasisi ya uchunguzi wa kiswahili, Kur. $1-24$.

Mwanzi, H. (1982). "Children's Literature in Kenya. An analysis of children's prose fiction." M. A. Thesis, University of Nairobi.

Mzandi, M. (2011), "Nafasi ya Utani Msibani na Harusini kwa Kabila la Wadigo".Ripoti ya Utafiti kwa Ajili ya Shahada Umahiri Chuo Kikuu cha Dodoma, (Haijachapishwa).

Ngugi, P. (2011). "Ufundishaji wa fasihi ya watoto katika shule za msingi Nchini Kenya." Katika kioo cha lugha, Juz. 9, Kur. 84-94.

Njogu, K. na Wafula, R. (2007). Nadharia za uhakiki wa fasihi. Nairobi: Jomo Kenyatta Foundation.

Njogu, K. na Chimerah, R; (1999): Ufundishaji wa Fasihi Nadharia na Mbinu. Nairobi: Jomo Kenyatta Foundation Ltd.

Ogacho, O. (1974), Grandparent-Grand Children Utani, URT, Vol.III, Dar es Salaam University Press.

Sengo, T.S. na Lucas (1975), Utani wa Jamii ya Wakwere, Nairobi Foundation Book Limited, Nairobi.
Tucker, N (1981). The child and the BOOK: A Psychological and Literary Exploration. Cambridge: Cambridge University Press.

TUKI (2004), Kamusi ya Kiswahili Sanifu, Oxford University Press, Nairobi

Wamitila, K. (2002). Uhakiki wa Fasihi: Misingi na vipengele vyake. Nairobi: Phoenix Publishers Ltd.

Wamitila, K. (2003 a). 'Influene or intertextuality? A comparative study of Kezilahabi's Nagona and Mzingile and Juan Ruifo's Pedro Paroma' Katika KISWAHILI vol. 66. Dar es Salaam.' TUKI. uk 49-58.

Wamitila, K. (2003). Kamusi ya Fasihi, Istilahi na Nadharia. Nairobi: Focus Books.

Wamitila, K. W. (2003a). Influence or intertextuality? A comparative study of Kezilahabi's Nagona and Mzingile and Juan Ruifo's Pedro Paroma'. Kiswahili, 66, 49-58.

Wanyela. S. (2007) Matumizi ya ushairi katika tamthilia ya Kiswahili. Tasnifu ya Uuzamili (Haijachapishwa). C, chuo kikuu cha Kenyatta (Haijachapishwa).

11 This work is licensed under a Creative Commons Attribution 4.0 International License. 